\title{
Fast Escaping Set of Transcendental Semigroup
}

\author{
Bishnu Hari Subedi*, Ajaya Singh \\ Central Department of Mathematics, Tribhuvan University, Kirtipur, Kathmandu, Nepal
}

Copyright (C) 2019 by authors, all rights reserved. Authors agree that this article remains permanently open access under the terms of the Creative Commons Attribution License 4.0 International License

\begin{abstract}
In this paper, we study fast escaping sets of transcendental semigroups. In particular, we discuss some fundamental structure and properties of fast escaping sets. We also show how far the classical dynamical theory of fast escaping sets of transcendental entire functions applies to general settings of transcendental semigroups and what new phenomena can occur.
\end{abstract}

Keywords Tanscendental semigroup, escaping set, fast escaping set, L-th levels of fast escaping set.

AMS Subject Classification (2010): 37F10, 30D05

\section{Introduction}

The principal aim of this paper is to study fast escaping sets not of single transcendental entire maps of complex plane $\mathbb{C}$, but of semigroups generated by families of such maps under composition. Let $\mathscr{F}$ be a collection of transcendental entire maps on $\mathbb{C}$. For any map $f \in \mathscr{F}$, the complex plane $\mathbb{C}$ is naturally partitioned into two subsets: the set of normality and its complement. The set of normality or Fatou set $F(f)$ of $f$ is the largest open set on which the iterates $f^{n}=f \circ f \circ \ldots \circ f$ where $n \in \mathbb{N}$, is a normal family. The complement of $F(f)$ is the Julia set $J(f)$. A maximally connected subset of $F(f)$ is a Fatou component. The escaping set of any $f \in \mathscr{F}$ is the set

$$
I(f)=\left\{z \in \mathbb{C}: f^{n}(z) \rightarrow \infty \text { as } n \rightarrow \infty\right\}
$$

and any $z \in I(S)$ is called escaping point. For any transcendental entire function $f$, the $I(f)$ was first studied by A. Eremenko [4]. He showed that $I(f) \neq \emptyset, J(f)=\partial I(f)$, $I(f) \cap J(f) \neq \emptyset$, and $\overline{I(f)}$ has no bounded component. In view of this last statement, he posed a conjecture:

Conjecture 1.1. Every component of $I(f)$ is unboun-ded.

This conjecture is considered an important open problem of transcendental dynamics, and nowadays it is famous as Eremenko's conjecture. This conjecture 1.1 has been proved for the fast escaping set $A(f)$, which consists of points that tend to infinity as fast as possible under iteration. This set is a subset of $I(S)$, and it was introduced first time by Bergweiler and Hinkkanen [3] and defined in the following form by Rippon and Stallard [15].

Definition 1.1. For a transcendental entire function $f$, the fast escaping set is defined by

$$
\begin{aligned}
& A(f)=\{z \in \mathbb{C}: \exists L \in \mathbb{N} \text { such that } \\
& \left.\qquad\left|f^{n+L}(z)\right| \geq M^{n}(R) \text { for } n \in \mathbb{N}\right\}
\end{aligned}
$$

where $M(r)=\max _{|z|=r}|f(z)|, r>0$ and $M^{n}(r)$ denotes $n t h$ iteration of $M(r)$ with respect to $r . R>0$ can be taken to be any number such that $M(r)>r$ for $r \geq R$.

Recall that

$$
C(f)=\left\{z \in \mathbb{C}: f^{\prime}(z)=0\right\}
$$

(where / represents derivative of $f$ with respect to $z$ ) is the set of critical points and

$$
C V(f)=\left\{w \in \mathbb{C}: w=f(z) \text { such that } f^{\prime}(z)=0\right\}
$$

is the set of critical values of $f$. The set $A V(f)$ consisting of all $w \in \mathbb{C}$ such that there exists a curve $\Gamma:[0, \infty) \rightarrow \mathbb{C}$ so that $\Gamma(t) \rightarrow \infty$ and $f(\Gamma(t)) \rightarrow w$ as $t \rightarrow \infty$ is the set of asymptotic values of $f$ and

$$
S V(f)=\overline{(C V(f) \cup A V(f))}
$$

is the set of singular values of $f$. If $S V(f)$ is finite, then $f$ is said to be of finite type. If $S V(f)$ is bounded, then $f$ is said to be of bounded type. The sets

$$
\mathscr{S}=\{f: f \text { is of finite type }\}
$$

and

$$
\mathscr{B}=\{f: f \text { is of bounded type }\}
$$

are respectively known as Speiser class and Eremenko- Lyubich class. The main concern of transcendental iteration theory is to describe the nature of the components of Fatou sets and the structure and properties of the Julia sets, escaping sets, 
Fast Escaping Set of Transcendental Semigroup

and fast escaping sets. We refer to the monograph: Dynamics of Transcendental Entire Functions [7] and to the book: Holomorphic Dynamics [11] for basic facts concerning the Fatou set, Julia set and escaping set of a transcendental entire functions. We refer to $[14,15,16]$ for facts and results concerning the fast escaping set of a transcendental entire functions.

Our particular interest is to study the dynamics of semigroups generated by a class of transcendental entire maps. For a collection $\mathscr{F}=\left\{f_{\alpha}\right\}_{\alpha \in \Delta}$ of such maps, let $S=\left\langle f_{\alpha}\right\rangle$ be a transcendental semigroup generated by them. The index set $\Delta$ is allowed to be infinite in general. Each $f \in S$ is a transcendental entire function, and $S$ is closed under functional composition. Thus, $f \in S$ is constructed through the composition of finite number of functions $f_{\alpha_{k}},(k=1,2,3, \ldots, m)$. That is, $f=f_{\alpha_{1}} \circ f_{\alpha_{2}} \circ f_{\alpha_{3}} \circ \cdots \circ f_{\alpha_{m}}$. A semigroup generated by finitely many transcendental entire functions $f_{i},(i=1,2, \ldots, n)$ is called finitely generated transcendental semigroup and we write $S=\left\langle f_{1}, f_{2}, \ldots, f_{n}\right\rangle$. If $S$ is generated by only one transcendental entire function $f$, then $S$ is a cyclic transcendental semigroup and we write $S=\langle f\rangle$. In this case, each $g \in S$ can be written as $g=f^{n}$. Note that in our study of semigroup dynamics, we say $S=\langle f\rangle$ is a trivial semigroup. The transcendental semigroup $S$ is abelian if $f_{\alpha} \circ f_{\beta}=f_{\beta} \circ f_{\alpha}$ for all generators $f_{\alpha}, f_{\beta}$ of $S$. The transcendental semigroup $S$ is bounded type (finite type) if each of its generators $f_{\alpha}$ is bounded type (finite type).

The family $\mathscr{F}$ of analytic maps forms a normal family in a domain $D$ if every sequence of the member of $\mathscr{F}$ contains a subsequence which converges or diverges locally uniformally in $D$. If there is a neighborhood $U$ of the point $z \in \mathbb{C}$ such that $\mathscr{F}$ is normal family in $U$, then we say $\mathscr{F}$ is normal at $z$.

Let $f$ be a transcendental entire map. We say that $f$ is iteratively divergent at $z \in \mathbb{C}$ if $f^{n}(z) \rightarrow \infty$ as $n \rightarrow \infty$. Semigroup $S$ is iteratively divergent at $z$ if $f^{n}(z) \rightarrow \infty$ as $n \rightarrow \infty$ for all $f \in S$. A semigroup $S$ is said to be iteratively bounded at $z$ if there is $f \in S$ which is not iteratively divergent of $z$. Note that in a semigroup $S=\left\langle f_{\alpha}\right\rangle$ if $f \in S$, then for all $m \in \mathbb{N}, f^{m} \in S$. So, $f^{m}=f_{\alpha_{1}} \circ f_{\alpha_{2}} \circ f_{\alpha_{3}} \circ \cdots \circ f_{\alpha_{p}}$ for some $p \in \mathbb{N}$. In this sense, any $f \in S$ is iteratively divergent at $z$ always means that there is a sequence $\left(g_{n}\right)_{n \in \mathbb{N}}$ in $S$ representing $g_{1}=f, g_{2}=f^{2}, \ldots, g_{n}=f^{n}, \ldots$ such that $g_{n}(z) \rightarrow \infty$ as $n \rightarrow \infty$. In this sense, semigroup $S$ is iteratively divergent at any $z \in \mathbb{C}$ if every sequence in $S$ has a subsequence which diverges to infinity at $z$.

Based on the Fatou-Julia-Eremenko theory of a transcedental entire function, the Fatou set, Julia set and escaping set of transcendental semigroup are defined as follows.

Definition 1.2 (Fatou set, Julia set and escaping set). Fatou set of the transcendental semigroup $S$ is defined by

$$
F(S)=\{z \in \mathbb{C}: S \text { is normal at } z\}
$$

and the Julia set $J(S)$ of $S$ is the compliment of $F(S)$. The escaping set of $S$ is defined by

$$
I(S)=\{z \in \mathbb{C}: S \text { is iteratively divergent at } z\} .
$$

We call each point of the set $I(S)$ by escaping point.
It is obvious that $F(S)$ is a largest open subset of $\mathbb{C}$ such that semigroup $S$ is normal. Hence its compliment $J(S)$ is a closed set. Whereas the escaping set $I(S)$ is neither an open nor a closed set (if it is non-empty). Any maximally connected subset $U$ of $F(S)$ is called a Fatou component. If $S=\langle f\rangle$, then $F(S), J(S)$ and $I(S)$ are respectively the Fatou set, Julia set and escaping set in classical complex dynamics. In this situation, we simply write $F(f), J(f)$ and $I(f)$.

In this paper, classical transcendental dynamics refers to the iteration theory of single transcendental map and transcendental semigroup dynamics refers to the dynamical theory generated by a set of transcendental entire maps. In transcendental semigroup dynamics, algebraic structure of semigroup naturally attached to the dynamics and hence the situation is complicated. The fundamental contrast between classical transcendental dynamics and semigroup dynamics appears by different algebraic structure of corresponding semigroups. In fact, nontrivial semigroup may not be abelian in general, however trivial semigroup is cyclic and therefore, abelian. As we discussed before, classical complex dynamics is a dynamical study of trivial (cyclic) semigroup whereas semigroup dynamics is a dynamical study of non-trivial semigroup.

\section{Motivation and Statement of the Pro- blem}

The main motivation of this paper comes from seminal work of Hinkkanen and Martin [6] on the dynamics of rational semigroups and the extension study of K. K. Poon [12] and Zhigang Haung [25] to the dynamics of transcendental semigroups. Both of them naturally generalized some results of classical complex dynamics to the semigroup dynamics as well as they also investigated some new phenomena in semigroup dynamical system. Another motivation of studying escaping sets and some extra properties and structure of Fatou sets and Julia sets of transcendental semigroups comes from the work of Dinesh Kumar and Sanjay Kumar [8, 9, 10] where they defined escaping set and discussed how far escaping set of classical transcendental dynamics can be generalized to semigroup dynamics. In parallel, we also studied certain structure and propperties of Fatou sets, Julia sets and escaping sets under semigroup dynamics in $[17,18,19,20,21,22,23,24]$. From these attempts, we again more motivated to the study of fast escaping sets of transcendental semigroups.

In this paper, we introduce fast escaping set in transcendental semigroup dynamics. The principal aim of this paper is to show how far classical transcendental dynamics applies to transcendental semigroup dynamics and what new phenomena appear in transcendental semigroup settings. Note that the fast escaping set $A(f)$ now plays a key role in classical transcendental dynamics which was introduced first time by Bergweiler and Hinkkanen [3] and studied in more depth by Rippon and Stallard [15]. The set $A(f)$ has some properties exactly similar to those of $I(f)$. For example $A(f) \neq \emptyset$, in fact, it is infinite set ([3, Lemma 2]), $\partial A(f)=J(f)([3$, Lemma 3]) and ([15, 
Theorem $5.1(\mathrm{~b})]), A(f) \cap J(f) \neq \emptyset([3$, Lemma 3]) and ([15, Theorem 5.1(a)]), The set $A(f)$ is completely invariant under $f([15$, Theorem $2.2(\mathrm{a})])$ and the set $A(f)$ does not depend on the choice of $R>0$ ([15, Theorem 2.2 (b)]). However, in [14, Theorem 1] and [15, Theorem 1.1], it is shown that all components of $A(f)$ are unbounded and since $A(f) \subset I(f)$ so it provides a partial answer to Eremenko's conjecture 1.1. If $U$ is a Fatou component of $f$ in $A(f)$, then its boundary is also in $A(f)$, that is, $\bar{U} \subset A(f)$ ([14, Theorem 2]) and ([15, Theorem 1.2]). These are much stronger properties of $A(f)$ than the set $I(f)$. In next sections 3, 4 and 5, we define and formulate fast escaping set in transcendental semigroup and we also examine how far these stated properties of classical transcendental dynamics can be generalized to semigroup dynamics.

\section{Fast Escaping Set of Transcendental Semigroup}

There is no formulation of fast escaping sets in transcendental semigroup dynamics. We have started to define fast escaping set and try to formulate some other related terms and results.

Let $S$ be a transcendental semigroup. Let us define a set

$$
\begin{aligned}
& A_{R}(S)=\left\{z \in \mathbb{C}:\left|f^{n}(z)\right| \geq M^{n}(R)\right. \\
& \qquad \text { for all } f \in S \text { and } n \in \mathbb{N}\}
\end{aligned}
$$

where $M(r)=\max _{|z|=r}|f(z)|$ and $M^{n}(r)$ denotes the nth iterates of $M(r) . R>0$ can be taken any value such that $M(r)>r$ for $r \geq R$. If $r$ is sufficiently large then $M^{n}(r) \rightarrow$ $\infty$ as $n \rightarrow \infty$. The set $A_{R}(S)$ is well defined in semigroup $S$ because for any $f \in S, f^{n} \in S$ for all $n \in \mathbb{N}$. From the condition $\left|f^{n}(z)\right| \geq M^{n}(R)$ for all $f \in S$ and $n \in \mathbb{N}$ of the set $A_{R}(S)$, we can also say that a point $z \in \mathbb{C}$ is in $A_{R}(S)$ if every sequence $\left(g_{n}\right)_{n \in \mathbb{N}}$ in $S$ has a subsequence $\left(g_{n_{k}}\right)_{n_{k} \in \mathbb{N}}$ which increases without bound at least as fast as the n-iterates of the maximum modulus of each $g_{n_{k}}$.

Definition 3.1 (Fast escaping set). The fast escaping set $A(S)$ of a transcendental semigroup $S$ consists the set $A_{R}(S)$ and all its pre-images. In other words, fast escaping set is a set of the form

$$
A(S)=\bigcup_{n \geq 0} f^{-n}\left(A_{R}(S)\right)
$$

where $f^{-n}\left(A_{R}(S)\right)=\left\{z \in \mathbb{C}: f^{n}(z) \in A_{R}(S)\right\}$ for all $f \in S$ and $n \in \mathbb{N}$.

We can do certain stratification of fast escaping set which helps to make it more visible and provides a significant new understanding of the structure and properties of this set. We can write fast escaping set as a countable union of all its labels as we define below.

Definition 3.2 ( $\mathrm{L}$ th label of fast escaping set). Let $A(S)$ be a fast escaping set of transcendental semigroup $S$. For $L \in \mathbb{Z}$, then the set of the form

$$
\begin{aligned}
& A_{R}^{L}(S)=\left\{z \in \mathbb{C}:\left|f^{n}(z)\right| \geq M^{n+L}(R)\right. \\
& \qquad \text { for all } f \in S, n \in \mathbb{N} \text { and } n+L \geq 0\}
\end{aligned}
$$

is called Lth level of fast escaping set $A(S)$.

Note that the set $A_{R}(S)$ defined above in 3.1 is the Oth level of $A(S)$. Since $M^{n+1}(R)>M^{n}(R)$ for all $n \geq 0$, so from 3.3 , we get following chain of relation

$$
\begin{gathered}
\ldots \subset A_{R}^{L}(S) \subset A_{R}^{L-1}(S) \subset \ldots \subset A_{R}^{1}(S) \subset A_{R}(S) \subset \\
A_{R}^{-1}(S) \subset A_{R}^{-2}(S) \subset \ldots \subset A_{R}^{-(L-1)}(S) \subset A_{R}^{-L}(S) \subset \ldots
\end{gathered}
$$

From 3.2 and 3.4, the fast escaping set can also be written as an expanding union of its labels.

$$
A(S)=\bigcup_{L \in \mathbb{N}} A_{R}^{-L}(S)
$$

Again from the definition 3.1, that is, from 3.2, if any $z_{0} \in$ $A(S)$, then $z_{0} \in f^{-n}\left(A_{R}(S)\right)$ for some $n \geq 0$. It gives $f^{n}\left(z_{0}\right) \in A_{R}(S)$ for all $f \in S$. From 3.1, there is $L \in \mathbb{N}$ such that $\left|f^{L}\left(f^{n}\left(z_{0}\right)\right)\right|=\left|f^{n+L}\left(z_{0}\right)\right| \geq M^{n}(R)$. With this clause, the fast escaping set of transcendental semigroup $S$ can now be written as

$$
\begin{aligned}
& A(S)=\{z \in \mathbb{C}: \text { there exists } L \in \mathbb{N} \text { such that } \\
& \left.\quad\left|f^{n+L}(z)\right| \geq M^{n}(R) \text { for all } f \in S, \text { and } n \in \mathbb{N}\right\} .
\end{aligned}
$$

We can use any one of the form 3.2 or 3.5 or 3.6 as a definition of fast escaping set. Note that by the definition (3.2 or 3.5 or 3.6), fast escaping set $A(S)$ of any transcendental semigroup $S$ is a subset of escaping set $I(S)$. Since from [17, Theorem 1.2 (3)], we can say that $I(S)$ may be empty. For any transcendental semigroup $S$, if $I(S)=\emptyset$, then we must have $A(S)=\emptyset$. It is not known whether there is a transcendental semigroup $S$ such that $I(S) \neq \emptyset$ but $A(S)=\emptyset$. Note that in classical transcendental dynamics, both of these sets are non-empty.

Example 3.1. Let $S$ be a transcendental semigroup generated by the functions $f(z)=e^{z}$ and $g(z)=e^{-z}$. Since $h=g \circ$ $f^{n} \in S$ is iteratively bounded at any $z \in \mathbb{C}$. So, $I(S)=\emptyset$ and $A(S)=\emptyset$.

Like escaping set $I(S)$, fast escaping set $A(S)$ is also neither an open nor a closed set if it is non-empty. Similar to the result [17, Theorem 1.2 (3)] of escaping set, the following result is also clear from the definition of fast escaping set.

Theorem 3.1. Let $S$ be a transcendental semigroup. Then $A(S) \subset A(f)$ for all $f \in S$ and hence $A(S) \subset \bigcap_{f \in S} A(f)$.

We have mentioned several results and examples of transcendental semigroups in [17, 18, 19, 21, 22, 23] where escaping set is non-empty. One of the latest particular result in 
the field of transcendental semigroup dynamics is a condition for which escaping set of the semigroup is same as the escaping set of its each function. In such case, the fact would be obvious from classical transcendental dynamics that the fast escaping set is also non-empty.

\section{Elementary Properties of Fast Esca- ping Set}

In this section, we check how far basic properties of fast escaping set of classical transcendental dynamics can be generalized to fast escaping set of transcendental semigroup dynamics. In [18], we examined the contrast between classical complex dynamics and semigroup dynamics in the invariant features of Fatou sets, Julia sets and escaping sets. In this paper, we see the same type of contrast in fast escaping set. Note that in classical transcendental dynamics, the fast escaping set is completely invariant.

Definition 4.1 (Forward, backward and completely invariant set). For a given semigroup $S$, a set $U \subset \mathbb{C}$ is said to be $S$-forward invariant if $f(U) \subset U$ for all $f \in S$. It is said to be $S$-backward invariant if $f^{-1}(U)=\{z \in \mathbb{C}: f(z) \in U\} \subset U$ for all $f \in S$ and it is called $S$-completely invariant if it is both $S$-forward and S-backward invariant.

We prove the following elementary results that are important regarding the structure of fast escaping set $A(S)$. These results may also have more chances of leading further results concerning the properties and structure of $A(S)$. Indeed, it shows certain connection and contrast between classical transcendental dynamics and transcendental semigroup dynamics and it is also a nice generalization of classical transcendental dynamics to semigroup dynamics.

Theorem 4.1. Let $S$ is a transcendental semigroup such that $A(S) \neq \emptyset$. Then the following are hold.

1. $A(S)$ is $S$-forward invariant.

2. $A(S)$ is independent of $R$.

3. $J(S)=\partial A(S)$.

4. $J(S) \subset \overline{A(S)}$.

5. $A(S) \cap J(S) \neq \emptyset$.

Proof. (1). From the definition 3.2 (that is, from equation 3.3), we can write

$$
A_{R}^{L}(S) \subset\left\{z \in \mathbb{C}:|z| \geq M^{L}(R), L \geq 0\right\} .
$$

So for any $z_{0} \in A_{R}^{L}(S)$,

$$
f\left(z_{0}\right) \in\left\{z \in \mathbb{C}:|f(z)| \geq M^{L+1}(R), L \geq 0\right\}=A_{R}^{L+1}(S)
$$

for all $f \in S$ and $n \in \mathbb{N}$. This shows that

$$
f\left(A_{R}^{L}(S)\right) \subset A_{R}^{L+1}(S)
$$

for all $f \in S$. However from relation 3.4,

$$
A_{R}^{L+1}(S) \subset A_{R}^{L}(S) .
$$

Hence, we have

$$
f\left(A_{R}^{L}(S)\right) \subset A_{R}^{L}(S) .
$$

This fact together with equation 3.5, we can say that $A(S)$ is S-forward invariant.

(2) Choose $R_{0}>R$, then from 3.4, we have

$$
A_{R_{0}}^{L}(S) \subset A_{R}^{L}(S)
$$

for all $L \in \mathbb{Z}$ and so

$$
\bigcup_{L \in \mathbb{N}} A_{R_{0}}^{-L}(S) \subset \bigcup_{L \in \mathbb{N}} A_{R}^{-L}(S)
$$

Since there is $m \in \mathbb{N}$ such that $M^{m}(R)>R_{0}$ and so

$$
\begin{aligned}
\bigcup_{L \in \mathbb{N}} A_{R}^{-L}(S) \subset & \bigcup_{L \in \mathbb{N}} A_{R}^{m-L}(S) \\
& =\bigcup_{L \in \mathbb{N}} A_{M^{m}(R)}^{-L}(S) \subset \bigcup_{L \in \mathbb{N}} A_{R_{0}}^{-L}(S)
\end{aligned}
$$

From above two inequality, we have

$$
\bigcup_{L \in \mathbb{N}} A_{R_{0}}^{-L}(S)=\bigcup_{L \in \mathbb{N}} A_{R}^{-L}(S)=A(S)
$$

This proves $A(S)$ is independent of $R$.

(3) We prove this statement by showing $(A(S))^{0} \subset F(S)$ and $(A(S))^{e} \subset F(S)$ where $(A(S))^{0}$ and $(A(S))^{e}$ are respectively interior and exterior of $A(S)$. Since $A(S)$ is Sforward invariant, so $f^{n}(A(S)) \subset A(S)$ for all $f \in S$ and $n \in \mathbb{N}$. Suppose $z \in(A(S))^{0}$, then there is a neighborhood $V$ of $z$ such that $z \in V \subset A(S)$. Since $A(S)$ contains no periodic points, so $\left|f^{n+L}(z)\right| \geq M^{n}(R)$ for all $f \in S$, and $n \in \mathbb{N}$ and hence $\left(f^{n}\right)_{n \in \mathbb{N}}$ is normal on $V$ by Montel's theorem. Thus $z \in F(S)$. This proves $(A(S))^{0} \subset F(S)$.

By the theorem 3.2.3 of [1], the closure and complement of $A(S)$ are also forward invariant. So from $f^{n}(A(S))$

$\subset A(S)$, we can write

$$
f^{n}(\mathbb{C}-\overline{A(S)}) \subset \mathbb{C}-\overline{A(S)} .
$$

for all $n \in \mathbb{N}$. Since $\mathbb{C}-\overline{A(S)}=(A(S))^{e}$. By the assumption of non-empty $A(S), \overline{A(S)}$ is also a non-empty closed set. By the definition, $F(S)$ is a largest open set on which $S$ is normal family, so we must

$$
\mathbb{C}-\overline{A(S)}=(A(S))^{e} \subset F(S)
$$

(4) The proof follows from (3).

(5) By the theorem 3.1, $A(S) \subset A(f)$ for all $f \in S$. A Fatou component $U \subset F(S)$ is also a component of $F(f)$ for each $f \in S$.

Case (i): If $U$ is multiply connected component of $F(S)$, then 
by [14, Theorem 2 (a)] $\bar{U} \subset A(f)$ for all $f \in S$. Again by the above same theorem 3.1, $\bar{U} \subset A(S)$. This shows that $\partial U \subset$ $A(S)$. Since $\partial U \subset J(f)$ for all $f \in S$. By [12, Theorem 4.2], we write $\partial U \subset J(S)$. This proves $A(S) \cap J(S) \neq \emptyset$.

case (ii): If $U$ is simply connected component of $F(S)$ that meets $A(S)$, then by [15, Theorem 1.2 (b)] $\bar{U} \subset A(f)$ for all $f \in S$. So, as in case (i), $\bar{U} \subset A(S)$. By [15, Corollary 4.6], if $F(S)$ has only simply connected components, then $\partial A_{R}^{L} \subset$ $J(S)$ where $\partial A_{R}^{L}(S)$ is L-th label of $F(S)$. From the equation 3.5 , we conclude that $A(S) \cap J(S) \neq \emptyset$.

There are many classes of functions from which we get $I(f) \subset J(f)$ and for such functions, we must have $A(f) \subset$ $J(f)$. Dinesh Kumar and Sanjay Kumar [8, Theorem 4.5] prove that $I(S) \subset J(S)$ if transcendental semigroup $S$ is of finite or bounded type. We prove the following similar result.

Theorem 4.2. Let $S$ be a bounded or finite type transcendental semigroup. Then $A(S) \subset J(S)$ and $J(S)=\overline{A(S)}$.

Proof. For each $f \in S$, Eremenko and Lyubich [5] proved that $I(f) \subset J(f)$. K. K. Poon [12, Theorem 4.2] proved that $J(S)=\overline{\bigcup_{f \in S} J(f)}$. So for any $f \in S$, we have $A(S) \subset$ $A(f) \subset J(f) \subset J(S)$. The second part follows from $A(S) \subset$ $J(S)$ together with above theorem 4.1 (4).

There are many functions in the class $\mathscr{B}$, the escaping set $I(f)$ consists of uncountable family of curves tending to infinity. For example, function $\lambda \sin z+\gamma$ with $\lambda, \gamma \in \mathbb{C}$ belongs to the class $\mathscr{S} \subset \mathscr{B}$ and its escaping set is an uncountable union of curves tending to infinity, the so-called Cantor bouquet. For the function $f(z)=\lambda e^{z}, 0<\lambda<1 / e$, the Fatou set is completely invariant attracting basin and Julia set is a Cantor bouquet consisting of uncountably many disjoint simple curves, each of which has finite end point and other endpoint is $\infty$. The escaping set of such a function consists of open curves (without endpoints) together with some of their end points. Note that for such a function, each point in the escaping set can be connected to $\infty$ by a curve in the escaping set. For such functions, every point in such a curve belongs to fast escaping set except possibly a finite endpoint. More generally, let $f$ be a finite composition of functions of finite order in the class $\mathscr{B}$ and let $z_{0} \in I(f)$. Then $z_{0}$ can be connected to $\infty$ by a simple curve $\Gamma \subset I(f)$ such that $\Gamma \backslash\left\{z_{0}\right\} \subset A(f)$ (see for instance [13, Theorem 1.2]).

There is a chance of similar result in semigroup dynamics if semigroup $S$ is generated by the transcendental functions of finite order in the class $\mathscr{B}$. If so, then every $f \in S$ is a finite composition of the functions of finite order in the class $\mathscr{B}$ and for each of such function $f, A(f)$ consists of curves $\Gamma \backslash\left\{z_{0}\right\}$ with exception of some of the end points. Since $A(S) \subset A(f)$ for each $f \in S$, then $A(S)$ may consist of curves $\Gamma \backslash\left\{z_{0}\right\}$ with exception of some of the end points.

\section{On the L-th Labels of $A(S)$}

In this section, we concentrate on L-th label $A_{R}^{L}(S)$ of fast escaping set $A(S)$. Since fast escaping set can be written as expanding union of L-th labels, so we hope that structure and properties of each L-th label may determine structure and properties of fast escaping set. Again, we will also see contrast between fast escaping set and its label if there are. The following result is a contrast. That is, analogous to classical transcendental dynamics [15], unlike the set $A(S)$, each of its label is a closed set.

Theorem 5.1. Let $L \in \mathbb{Z}$, then for a transcendental semigroup $S$ such that $A(S) \neq \emptyset$. Then the set $A_{R}^{L}(S)$ is closed and unbounded for each $L \in \mathbb{Z}$ if it is non-empty.

Proof. From the definition 3.2, we can write

$$
A_{R}^{L}(S) \subset A_{R}^{L}(f)
$$

for all $f \in S$. This implies that

$$
A_{R}^{L}(S) \subset \bigcap_{f \in S} A_{R}^{L}(f)
$$

Since for each $L \in \mathbb{Z}, A_{R}^{L}(f)$ is a closed and unbounded set and also by [15, Theorem 1.1] each component of $A_{R}^{L}(f)$ is closed and unbounded for all $f \in S$. So, $\bigcap_{f \in S} A_{R}^{L}(f)$ is also a closed and unbounded set and each of its component is closed and unbounded as well. Since $A_{R}^{L}(S)$ is a component of $\bigcap_{f \in S} A_{R}^{L}(f)$, so it must be closed and unbounded.

On the light of this theorem 5.1 and equation 3.5, we have obtained a new structure of fast escaping set $A(S)$, a countable union of closed and unbounded sets $A_{R}^{L}(S)$. This result also provides a solution of Eremenko's conjecture 1.1 in transcendental semigroup dynamics. This generalizes the result of classical transcendental dynamics to transcendental semigroup dynamics.

Labels of fast escaping set $A(S)$ can be used to show if $U$ is a Fatou component in $A(S)$, then boundary of $U$ is also in $A(S)$. There are variety of results on simply connected and multiply connected Fatou components. Each of the Fatou component of transcendental semigroup is either a stable (periodic) or unstable (wandering (non- periodic)) domain as defined below.

Definition 5.1 (Stabilizer, wandering component and stable domains). For a transcendental semigroup $S$, let $U$ be a component of the Fatou set $F(S)$ and $U_{f}$ be a component of Fatou set containing $f(U)$ for some $f \in S$. The set of the form

$$
S_{U}=\left\{f \in S: U_{f}=U\right\}
$$

is called stabilizer of $U$ on $S$. If $S_{U}$ is non-empty, we say that a component $U$ satisfying $U_{f}=U$ is called stable basin for $S$. The component $U$ of $F(S)$ is called wandering if the set $\left\{U_{f}: f \in S\right\}$ contains infinitely many elements. That is, $U$ is a wandering domain if there is sequence of elements $\left\{f_{i}\right\}$ of $S$ such that $U_{f_{i}} \neq U_{f_{j}}$ for $i \neq j$. Furthermore, the component $U$ of $F(S)$ is called strictly wandering if $U_{f}=U_{g}$ implies $f=g$. A stable basin $U$ of a transcendental semigroup $S$ is

1. attracting if it is a subdomain of attracting basin of each $f \in S_{U}$ 
2. supper attracting if it is a subdomain of supper attracting basin of each $f \in S_{U}$

3. parabolic if it is a subdomain of parabolic basin of each $f \in S_{U}$

4. Siegel if it is a subdomain of Siegel disk of each $f \in S_{U}$

5. Baker if it is a subdomain of Baker domain of each $f \in$ $S_{U}$

Note that stabilizer $S_{U}$ is a a subsemigroup of $S$ ([22, Lemma 2.2]). Also, in classical case, a stable basin is one of above type. For any Fatou component $U$, we prove the following result which is analogous to [15, Theorem 1.2] of classical transcendental dynamics.

Theorem 5.2. Let $U$ be a Fatou component of transcendental semigroup $S$ that meets $A_{R}^{L}(S)$, where $R>0$ be such that $M(r, f)>r$ for $r \geq R$ for all $f \in S$ and $L \in \mathbb{N}$. Then

1. $\bar{U} \subset A_{R}^{L-1}(S)$;

2. if $U$ is simply connected, then $\bar{U} \subset A_{R}^{L}(S)$

Proof. Since $U \cap A_{R}^{L}(S) \neq \emptyset$. The fact $A_{R}^{L}(S) \subset A_{R}^{L}(f)$ for all $f \in S$ implies that $U \cap A_{R}^{L}(f) \neq \emptyset$ for all $f \in S$. So, from the theorem [15, Theorem 1.2 (a)], we always have $\bar{U} \subset A_{R}^{L-1}(f)$ for all $f \in S$. So $\bar{U} \subset A_{R}^{L-1}(S)$. The second part also follows similarly by using [15, Theorem 1.2 (b)].

By part (2) of above theorem 3.1, we can conclude that $\bar{U} \subset A_{R}^{L}(S)$ for all simply connected component $U$ of $F(S)$. So, if all components of $F(S)$ are simply connected, then we must $\partial A_{R}^{L}(S) \subset J(S)$ and hence interior of $A_{R}^{L}(S)$ is contained in $F(S)$. This theorem also generalizes the result of classical transcendental dynamics to transcendental semigroup dynamics. That is, whatever Fatou component (simply or multiply connected) $U$ of $F(S)$ intersecting $A(S)$, there is always $\bar{U} \subset A(S)$. Again, another question may raise. Such a Fatou component $U$ is periodic or wandering? Note that in classical transcendental dynamics, such a Fatou component is always wandering ([15, Corollary 4.2]). For transcendental semigroup dynamics, such a Fatou component is again wandering domain. For, if $U \cap A(S) \neq \emptyset$, then $U \cap A(f) \neq \emptyset$ for all $f \in S$. In this case $U$ is wandering domain of each $f \in S$, so it is wandering domain of $S$ as well.

In above paragraph, we discussed about a Fatou component intersecting the fast escaping set $A(S)$. Are there any Fatou components that lie in $A(S)$ ? In classical transcendental dynamics, its answer is yes (see for instance [15, Theorem 4.4] and [14, Theorem 2]). Indeed, in such case, the Fatou component that lie in $A(S)$ is a (closure of) multiply connected wandering domain. Bergweiler constructed an example of transcendental entire function $f$ for which $A(f)$ contains simply connected wandering domain ([2, Theorem 2]). This wandering domain is simply connected bounded one which lie in between multiply connected wandering domains and this one is only known example of non-multiply connected Fatou component that lie in $A(f)$. The generalization of above discussion to semigroup dynamics is also possible. For example, if $U$ is a multiply connected wandering domain of $F(S)$, then it is also multiply connected wandering domains of every $f \in S$. In this case, $\bar{U} \subset A(f)$ for all $f \in S([15$, Theorem 4.4]). Hence $\bar{U} \subset A(S)$.

\section{Conclusion and Final Remarks}

We defined fast escaping set in transcendental semigroup dynamics and we described some elementary properties. We showed that most of the properties of fast escaping set of classical transcendental dynamics are generalized to semigroup dynamics and few new phenomena were also occurred. In addition, there are some more things of classical transcendental dynamics regarding the structure (such as spider's web and Cantor bouquet) of fast escaping set (see for instance in $[14,15,16]$ ) which are not still formulated in semigroup dynamics. We will continue our study in this direction in coming days. Also, we will investigate some concrete examples of transcendental semigroups that have non empty fast escaping sets with additional visible structure and properties.

\section{Acknowledgements}

We are very grateful to experts, reviewers and referees for their appropriate and constructive suggestions to improve this paper.

We are very grateful to Prof. G. B. Thapa, Tribhuvan University, Kathmandu, Nepal for his thorough reading over the manuscript with fruitful discussion as well as valuable suggestions and comments.

We are also thankful to University Grants Commission, Nepal for PhD faculty fellowship and Research Support Programs of the HERP (Programm No-9, Research Article Publication) (2014-2020).

\section{REFERENCES}

[1] A. F. Beardon. Iteration of rational functions, Complex analytic dynamical systems, Graduate Texts in Mathematics 132, Spinger-Verlag, New York, Inc, 1991.

[2] W. Bergweiler. An entire functions with simply and multiply connected wandering domains, Pure Appl. Math. Quarterly, Vol. 7, 107-120, 2011

[3] W. Bergweiler, A. Hinkkanen. On semi-conjugations of entire functions, Math. Proc. Camb. Phil. Soc. Vol. 126, No. 3, 565574, 1999.

[4] A. Eremenko. On the iterations of entire functions, Dynamical System and Ergodic Theory, Banach Center Publication, Polish 
Scientific Publishers, Vol. 23, Warsaw, Poland, 339-344, 1989.

[5] A. Eremenko, M.Y. Lyubich. Dynamical Properties of Some Classes of Entire Functions, Ann. Inst. Fourier, Grenoble, Vol. 42, 989-1020, 1992.

[6] A. Hinkkanen, G. J. Martin. The dynamics of semigroups of rational functions- I, Proc. London Math. Soc. (3) 73, 358-384, 1996.

[7] X. H. Hua, C. C. Yang. Dynamic of transcendental functions, Gordon and Breach Science Publication, 1998.

[8] D. Kumar, S. Kumar. The dynamics of semigroups of transcendental entire functions-II, arXiv: 1401.0425 v3 [math.DS], 22 May 2014

[9] D. Kumar, S. Kumar. Escaping set and Julia set of transcendental semigroups, arXiv:141.2747 v3 [math. DS] 10 October, 2014

[10] D. Kumar, S. Kumar. Semigroup of transcendental entire functions and their dynamics, Proc. Indian Sci. (Math. Sci.) Vol. 127, No. 2, 349-360, 2017.

[11] S. Morosawa, Y. Nishimura, M. Taniguchi, T. Ueda. Holomorphic Dynamics, Cambridge University Press, Cambridge, UK, 2000.

[12] K. K. Poon. Fatou-Julia theory on transcendental semigroups, Bull. Austral. Math. Soc. Vol. 58, 403-410, 1998.

[13] L. Rempe, P. J. Rippon, G.M. Stallard. Are Devaney hairs fast escaping?, Diff. eq. and Appl., Vol. 16, 739-762, 2010.

[14] P. J. Rippon, G. M. Stallard. On the questions of Fatou and Eremenko, Proc. Amer. Math. Soc. Vol. 133, 1119-1126, 2005.

[15] P. J. Rippon, G. M. Stallard. Fast escaping points of entire functions, Proc. London Math. Soc. Vol. 3, No 4, 787-820,
2012.

[16] D. J. Sixsmith. Topics in transcendental dynamics, PhD Thesis, The Open University, UK, 2013.

[17] B. H. Subedi, A. Singh. A short comparison of classical complex dynamics and holomorphic semigroup dynamics, arXiv: 1803.08368v1 [math. DS], 22 March 2018.

[18] B. H. Subedi, A. Singh. Invariant properties of Fatou set, Julia set and escaping set of holomorphic semigroup, arXiv: 1803.09662v1 [math. DS], 23 March 2018.

[19] B. H. Subedi, A. Singh. Escaping set of hyperbolic semigroup, arXiv: 1803.10381v1 [math DS], 28 March 2018.

[20] B. H. Subedi, A. Singh. On completely invariant Julia set of transcendental semigroup, arXiv: 1804. 03554v1 [math. DS], 10 April 2018.

[21] B. H. Subedi, A. Singh. Completely invariant escaping set of transcendental semigroup, arXiv: 1804. 11252v1 [math.DS], 30 April 2018.

[22] B. H. Subedi, A. Singh. Fatou set, Julia set and escaping set in holomorphic subsemigroup dynamics, arXiv:1807.04499v1 [math. DS], 12 July 2018.

[23] B. H. Subedi, A. Singh. A study of Fatou Set, Julia set and escaping set in nearly abelian transcendental semigroup, arXiv: 1808. 00857v1 [math. DS], 1 August 2018.

[24] B. H. Subedi, A. Singh. A study of Fatou Set, Julia set and escaping set in conjugate transcendental semigroup, arXiv: 1808.03275v1 [math DS], 9 August 2018.

[25] H. Zhigang. The dynamics of semigroup of transcendental meromorphic functions, Tsinghua and Technology, Vol. 9, No. 4, 472-474, 2004. 